\title{
NEWS
}

\section{Scientists summit at Shanghai in the field of polymer crystallization}

\author{
Wenbing HU (凶) \\ Department of Polymer Science and Engineering, School of Chemistry and Chemical Engineering, \\ Nanjing University, Nanjing 210093, China
}

From Aug. 12 to 15 at Galaxy Hotel, Shanghai, more than 100 scientists and graduate students from macromolecular physics, engineering and chemistry met together in the International Discussion Meeting of Polymer Crystallization (IDMPC). The participants were coming from China, Japan, United States, Germany, England, France, Netherlands, Italy, Korea and Canada. The meeting was initiated by the Ministry of Education of China and European Physical Society, sponsored by the National Natural Science Foundation of China and Donghua University, and organized by the State Key Laboratory for Modification of Chemical Fibers and Chemical Materials.

The attendants brought the cutting-edge knowledge on polymer crystallization. Thirty oral presentations were given, covering various issues in polymer crystallization including the Avrami equation, flow-induced crystallization, functional polymers, nano-composites, semi-crystalline phases, crystal morphology, crystal reorganization, microscopic observations, advanced experiments and modeling. Following them were hot but friendly discussions. New results from computer simulations and theoretical evaluations, together with novel experimental observations, were communicated to stimulate new viewpoints, either following or in conflict with conventional wisdom. Particular emphasis were given to the following topics: (1) crystallization/melting of bio-sourced polymers; (2) new insight into polymer crystallization and melting caused by the chain-like nature of polymers, such as nucleation, precursor in oriented states, metastable crystals, re-organisation processes, and annealing; (3) new observations on polymer crystallization under space confinement, including thinfilms, nano-pore, droplets, microdomains of block copolymers; (4) new approaches (methodology and techniques) in the study of polymer crystallization and melting; (5) relation between lamellar thickness and the melting temperature.

Fifty-four posters were presented in the poster session; ten of them received the excellent poster awards. The awards were established to encourage youngsters to devote their career in the field of polymer crystallization. In the excursion of the meeting, all the participants visited the Shanghai Synchrotron Source. The new equipments impress the visitors as a sign of China's scientific research strength and facilitate an intensified collaboration in future research activities. 